\title{
Some Observations Regarding the SAFT-VR-Mie Equation of State
}

\author{
Vladimir Kalikhman ${ }^{1,2}$, Daniel Kost ${ }^{2}$ and Ilya Polishuk ${ }^{1, *}$ \\ ${ }^{I}$ Department of Chemical Engineering \& Biotechnology, Ariel University Center, 40700, Ariel, Israel \\ ${ }^{2}$ Department of Chemistry, Ben-Gurion University of the Negev, Beer-Sheva, 84105, Israel
}

\begin{abstract}
This study demonstrates that the advanced theoretical basis and the consequential numerical complexity do not always guarantee the success of EOS models in predicting the experimental thermodynamic property data. Although one of the best versions of SAFT, namely SAFT-VR-Mie might have doubtless advantages in predicting the data of nonspherical molecules, once again it is shown that there is a price to pay for the excessive model's complexity. In particular, the present study reveals a previously unnoticed kind of numerical pitfalls, yet generated by the chain term of the SAFTVR-Mie EOS. A possible way of avoiding the numerical pitfall under consideration is proposed.
\end{abstract}

Keywords: Equation of state, statistical association fluid theory, high pressure, phase equilibria, sound velocity, heat capacity.

\section{INTRODUCTION}

Equations of State (EoS) based on the Statistical Association Fluid Theory (SAFT) present a new generation of fluid phase models offering doubtless advantages over the popular cubic equations [1-5]. The SAFT with attractive potentials of Variable Range and Mie's monomer hard-core potential (SAFT-VR-Mie) [6] is one of the most successful versions of SAFT due to its advantages in predicting the auxiliary properties [7-10]. In addition, previously [11] it has been concluded that SAFT-VR-Mie is free of the fictitious phase equilibria numerical pitfall characteristic for several other versions of SAFT [12-15]. The current study aims answering two questions:

1 Is the success of SAFT-VR-Mie achieved thanks to its advanced theoretical basis or rather the appropriate parameters fitting?

2 Is this model indeed entirely free of numerical pitfalls?

Since the expression of SAFT-VR-Mie is sophisticated, some possible printing errors have appeared in the previous publications. Therefore it seems worthwhile to provide here its brief description.

\section{THEORY}

For the non-polar compounds SAFT models present the residual Helmholtz's energy as a sum of the following contributions:

$A^{\text {res }}=A^{H S}+A^{\text {disp }}+A^{\text {chain }}$

The hard-sphere (HS) contribution is given as follows:

*Address correspondence to this author at the Department of Chemical Engineering \& Biotechnology, Ariel University Center, 40700, Ariel, Israel; Tel: +972-3-9066346; Fax: +972-3-9066323;

E-mails: polishuk@ariel.ac.il, polishyk@bgumail.bgu.ac.il
$A^{H S}=m R T \frac{4 \eta-3 \eta^{2}}{(1-\eta)^{2}}$

where $\eta$ the packing fraction:

$\eta=\frac{\pi N_{A v}}{6 V} m \sigma^{3} \theta(T)$

$N_{A v}$ is the Avogadro's number, $m$ is the effective number of segments, $\sigma$ is the Lennard-Jones's segment diameter. Both $m$ and $\sigma$ are the model's adjustable parameters. According to SAFT-VR-Mie [6]:

$\theta(T)=\left(\begin{array}{l}0.995438-0.0259917(T \mathrm{k} / \varepsilon)+ \\ 0.00392254(T \mathrm{k} / \varepsilon)^{2}-0.000289398(T \mathrm{k} / \varepsilon)^{3}\end{array}\right)^{3}$

$\varepsilon$ is the inter-segment interaction's dispersion energy, $k$ is the Boltzmann's constant. $\varepsilon / k$ is the $3^{\text {rd }}$ model's adjustable parameter. It should be pointed out that the References [7-9] have used instead of Equation (4) the numerically calculated integral definition, a technique which is not completely clear to us. Nevertheless, it should be pointed out that since we were able to exactly reproduce all the results of the Reference [7], implementation of Equation (4) seems justified. Moreover, the temperature dependence of $\eta$ has no impact on the numerical pitfall discussed below.

The SAFT-VR-Mie's dispersion contribution is given as:

$A^{d i s p}=m R T\left(\beta a_{1}^{M}+\beta^{2} a_{2}^{M}\right)$

where

$\beta=\frac{1}{k T}$

The first perturbation term used in the previous study is given as: 


$$
a_{1}^{M}=C\left[a_{1}^{S}\left(\lambda_{1}\right)-a_{1}^{S}\left(\lambda_{2}\right)\right]
$$

$\lambda_{1}$ is typically equal to 6 and $\lambda_{2}$ is the $4^{\text {th }}$ model's adjustable parameters. The second perturbation term is:

$a_{2}^{M}=\frac{C}{2} \varepsilon \frac{(1-\eta)^{4}}{1+4 \eta+4 \eta^{2}} \eta \frac{\partial a_{1}^{S}\left(2 \lambda_{1}\right)}{\partial \eta}$

The expression corresponding to the mean attractive energy for a Sutherland- $\lambda$ system is:

$a_{1}^{S}\left(\lambda_{X}\right)=-\left.4 \eta \varepsilon\left(\frac{3}{\lambda_{i}-3}\right) \frac{1-\eta_{e f f} / 2}{\left(1-\eta_{e f f}\right)^{3}}\right|_{X=1,2}$

and the effective packing fraction is defined as:

$\eta_{\text {eff }}\left(\lambda_{X}\right)=c_{1} \eta+c_{2} \eta^{2}$

while $c_{1}$ and $c_{2}$ are given by the following matrix:

$\left(\begin{array}{l}c_{1} \\ c_{2}\end{array}\right)=\left.\left(\begin{array}{cccc}-0.943973 & 0.422543 & -0.0371763 & 0.00116901 \\ 0.370942 & -0.173333 & 0.0175599 & -0.000572729\end{array}\right)\left(\begin{array}{c}1 \\ \lambda_{x} \\ \lambda_{x}^{2} \\ \lambda_{x}^{3}\end{array}\right)\right|_{x=1,2}$

$a_{1}^{S}\left(2 \lambda_{1}\right)$ in Equation (8) means than $\lambda_{1}$ in Equations (9-11) is replaced by $2 \lambda_{1}$.

And, finally,

$\mathrm{C}=\frac{\lambda_{2}}{\lambda_{2}-\lambda_{1}}\left(\frac{\lambda_{2}}{\lambda_{1}}\right)^{\lambda_{1} /\left(\lambda_{2}-\lambda_{1}\right)}$

Some confusion appears concerning the SAFTVR-Mie's chain contribution. In particular, in the Reference [7] it is given as:

$A^{\text {chain }}=R T(1-m) \ln$

$\left[\frac{1-\eta / 2}{(1-\eta)^{3}}+\frac{\beta}{4}\left\{\frac{\partial a_{1}^{M}}{\partial \eta}-\frac{C \lambda_{1}}{4 \eta} a_{1}^{S}\left(\lambda_{1}\right)+\frac{C \lambda_{2}}{4 \eta} a_{2}^{S}\left(\lambda_{2}\right)\right\}\right]$

In the Reference [9] the last term of the expression above appears as:

$A^{\text {chain }}=R T(1-m) \ln$

$\left[\frac{1-\eta / 2}{(1-\eta)^{3}}+\frac{\beta}{4}\left\{\frac{\partial a_{1}^{M}}{\partial \eta}-\frac{C \lambda_{1}}{4 \eta} a_{1}^{S}\left(\lambda_{1}\right)+\frac{C \lambda_{2}}{4 \eta} a_{1}^{S}\left(\lambda_{2}\right)\right\}\right]$

Unfortunately, both equations (13a) and (13b) do not yield accurate modeling of data with the parameters listed in Reference [7]. However we were able to exactly reproduce the results of the Reference [7] with:

$A^{\text {chain }}=R T(1-m) \ln$

$\left[\frac{1-\eta / 2}{(1-\eta)^{3}}+\frac{\beta}{4}\left\{\frac{\partial a_{1}^{M}}{\partial \eta}-\frac{C \lambda_{1}}{3 \eta} a_{1}^{S}\left(\lambda_{1}\right)+\frac{C \lambda_{2}}{3 \eta} a_{1}^{S}\left(\lambda_{2}\right)\right\}\right]$
It should be pointed out that substitution Equations (6)(12) into Equations (5) and (13) results in the particularly long and complicated expressions. In the current study the performance of SAFT-VR-Mie is compared with the much simpler version of SAFT, namely the original SAFT of Chapman et al. [16]. This version implements the same expression for $A^{H S}$ however:

$\theta(T)=\left(\frac{1+0.2977(k / \varepsilon) T}{1+0.33163(k / \varepsilon) T+\left(0.0010477+0.025337 \frac{m-1}{m}\right)(k / \varepsilon)^{2} T^{2}}\right)^{3}$

$A^{d i s p}=m R(\varepsilon / k)\left(a_{1}^{M}+\frac{a_{2}^{M}(\varepsilon / k)}{T}\right)$

The original Chapman's et al. [16] expressions for $a_{1}^{M}$ and $a_{2}^{M}$ might be reduced to:

$$
\begin{aligned}
& a_{1}^{M}=\frac{3 \sqrt{2}}{\pi}\left[-8.5959 \eta-6.1344 \eta^{2}-3.87882 \eta^{3}+25.3316 \eta^{4}\right] \\
& a_{2}^{M}=\frac{3 \sqrt{2}}{\pi}\left[-1.9075 \eta+13.4675 \eta^{2}-40.5171 \eta^{3}+39.1711 \eta^{4}\right]
\end{aligned}
$$

and the chain term [16] is given as:

$A^{\text {chain }}=R T(1-m) \ln \frac{1-\eta / 2}{(1-\eta)^{3}}$

Having the expression for $A^{\text {res }}$, the pressure is obtained as:

$P=\frac{R T}{V}-\left(\frac{\partial A^{\text {res }}}{\partial v}\right)_{T}$

The residual heat capacities might be calculated using the following relationships:

$C_{V}^{r e s}=-T\left(\frac{\partial A^{r e s^{2}}}{\partial^{2} T}\right)_{v}$

$C_{P}^{r e s}=C_{V}^{r e s}-R-T \frac{(\partial P / \partial T)_{v}^{2}}{(\partial P / \partial v)_{T}}$

The values of $C_{V}$ and $C_{P}$ are obtained by adding the pertinent ideal gas properties available in the literature [17]. The sound velocities, enthalpies, entropies and the virial coefficients might be evaluated with:

$W=\sqrt{-\frac{C_{P}}{C_{V}} \frac{v^{2}}{M_{w}}\left(\frac{\partial P}{\partial v}\right)_{T}}$

$H^{\text {res }}=R T\left\{-T\left(\frac{\partial\left(A^{\text {res }} / R T\right)}{\partial T}\right)_{v}+\frac{P v}{R T}-1\right\}$ 


$$
\begin{aligned}
& S^{\text {res }}=R\left\{-T\left(\frac{\partial\left(A^{\text {res }} / R T\right)}{\partial T}\right)_{v}-\frac{A^{\text {res }}}{R T}+\ln \frac{P v}{R T}\right\} \\
& B=\operatorname{Lim}_{\rho \rightarrow 0}\left(\frac{\partial Z}{\partial \rho}\right)_{T} \\
& C=\frac{\operatorname{Lim}_{\rho \rightarrow 0}\left(\frac{\partial Z^{2}}{\partial^{2} \rho}\right)_{T}}{2}
\end{aligned}
$$

The calculations have been performed using the Mathematica $7^{\circledR}$ software (the pertinent routines can be obtained from the corresponding author by request).

\section{RESULTS}

In order to evaluate the contribution of the very sophisticated Equations (5) and (13) to the accuracy of SAFT-VR-Mie let as compare its performance with the much simpler original Chapman's et al. SAFT [16]. With the purpose of reducing the effect of the parameters fitting the following strategy is proposed:

1 The equal values of the parameters $m$ and $\sigma$ for both SAFTs are taken from the reference [7]. Then the nearly equal contributions of the HS terms are obtained and the deviations are originated mainly by the differences in the dispersion and the chain terms.

2 The Chapman et al. SAFT's $\varepsilon / k$ is fitted to the critical temperature yielded by SAFT-VR-Mie in order to achieve similarity between the phase envelopes predicted by both models.

Thus for methane the Chapman et al. SAFT's parameters are: $m=1, \sigma=3.7332 \AA$ and $\varepsilon / k=147.736 \mathrm{~K}$. The values of SAFT-VR-Mie's parameters are obtained from Reference [7]. Fig. (1) depicts the pressure contributions of the different SAFT's terms (the chain contribution is yet zero) at $100 \mathrm{~K}$. It should be pointed out that at different temperatures the same picture is obtained. In particular, it can be seen that the SAFT-VR-Mie's dispersion contribution is a bit smaller having only minor influence on the final result. In other words, both models predict the data in a similar manner. Moreover, it might be seen that the SAFT of Chapman et al. in spite of its simplicity is somewhat advantageous in predicting certain properties such as the vapour pressures, the high pressure and temperature isobaric heat capacities and the third virial coefficient (see Figs. 2-5). At the same time, SAFT-VR-Mie is more accurate in predicting the liquid isochoric heat capacities. Nevertheless it should be concluded that in the current case the deep theoretical approach and the excessively complex expressions do not provide major advantage in predicting the experimental data. In addition, it would be rather unlikely to reach substantially different conclusions in the cases of other non-polar spherical molecules. Thus, in what follows let us consider an example of the non-spherical molecule, namely ethane.

The Chapman et al. SAFT's parameters for ethane evaluated in the current study as: $m=1.33, \sigma=3.8741 \AA$ and $\varepsilon / k=200.222 \mathrm{~K}$. Fig. (6) depicts the pressure contributions of the different SAFT's terms. It might be seen that once

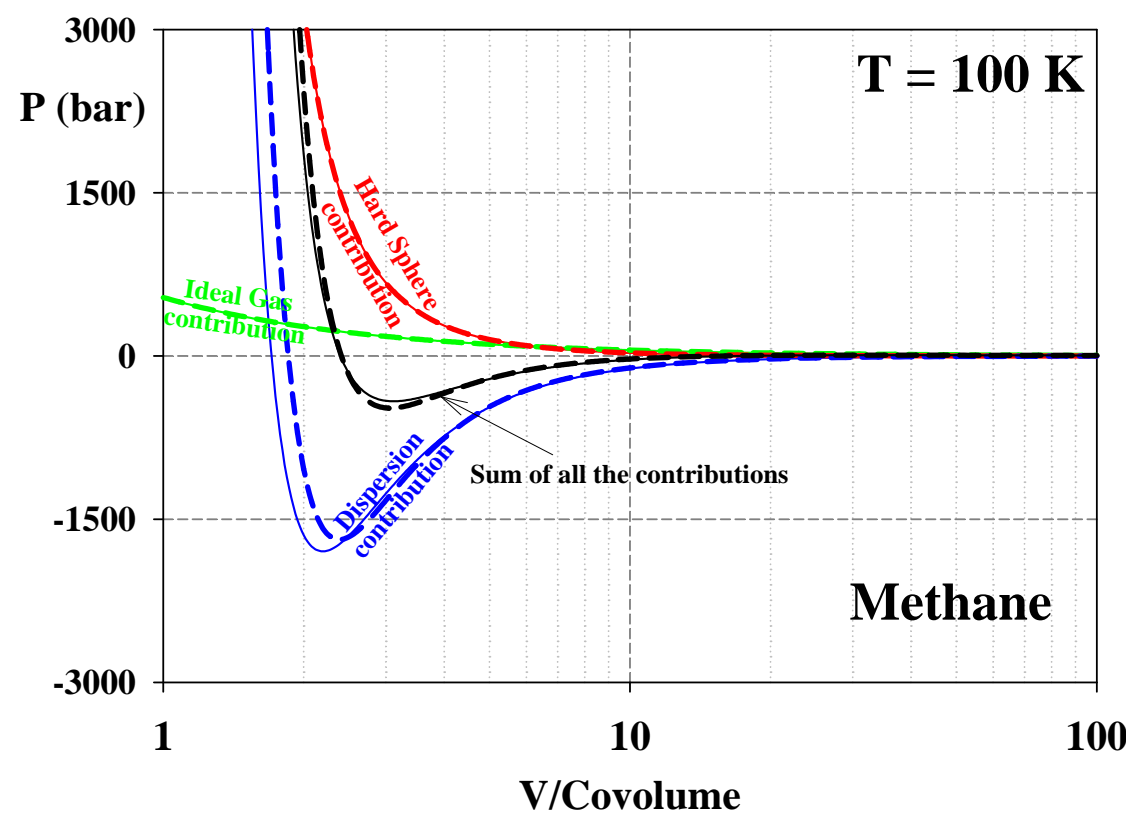

Fig. (1). Pressure contributions of different SAFT's parts for methane at $100 \mathrm{~K}$. Solid lines - SAFT-VR-Mie. Dotted lines - SAFT of Chapman's et al. 

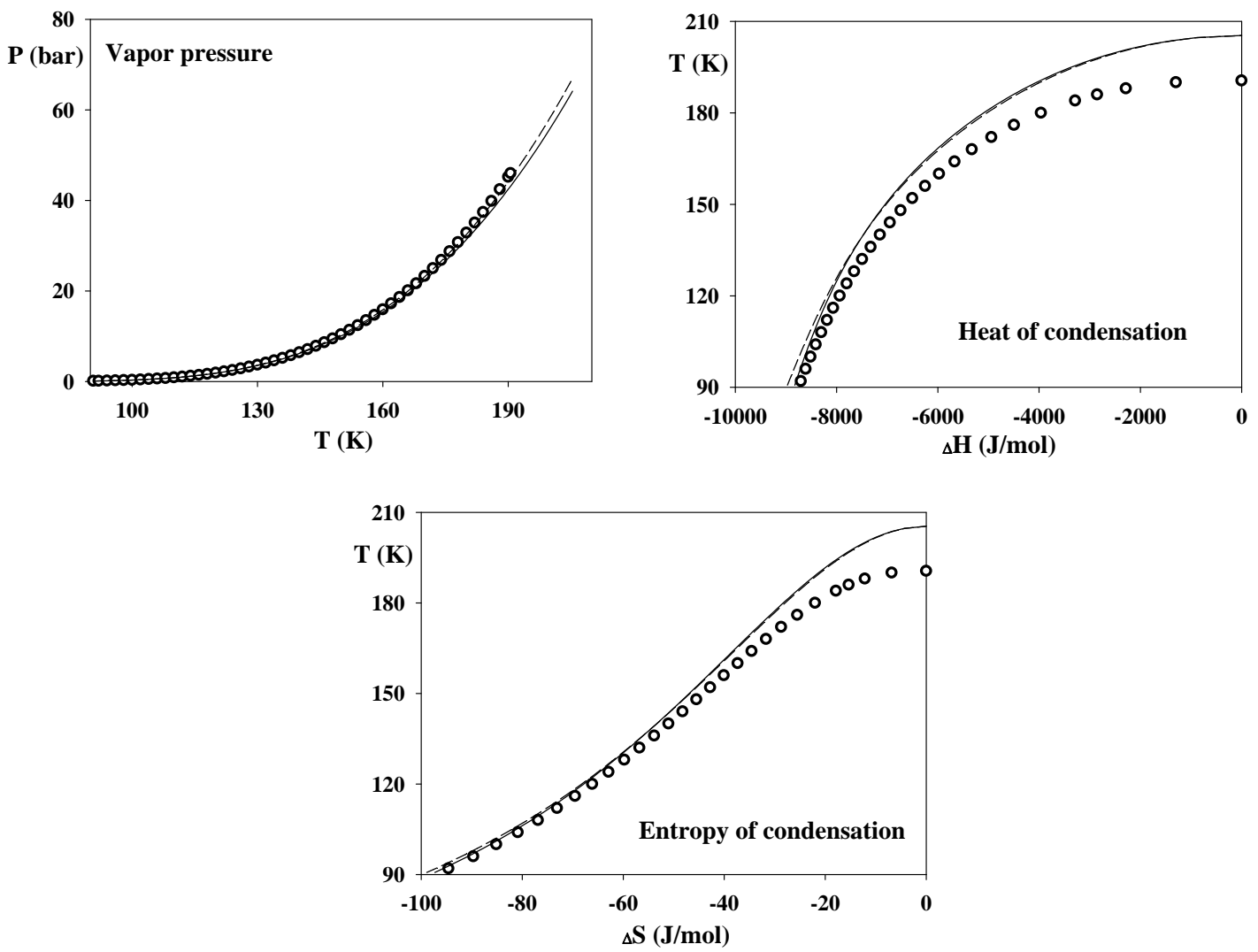

Fig. (2). Vapor pressure, enthalpy and entropy of condensation of methane. $\circ$ - experimental data [18]. Solid lines - SAFT-VR-Mie; dotted lines - SAFT of Chapman et al.
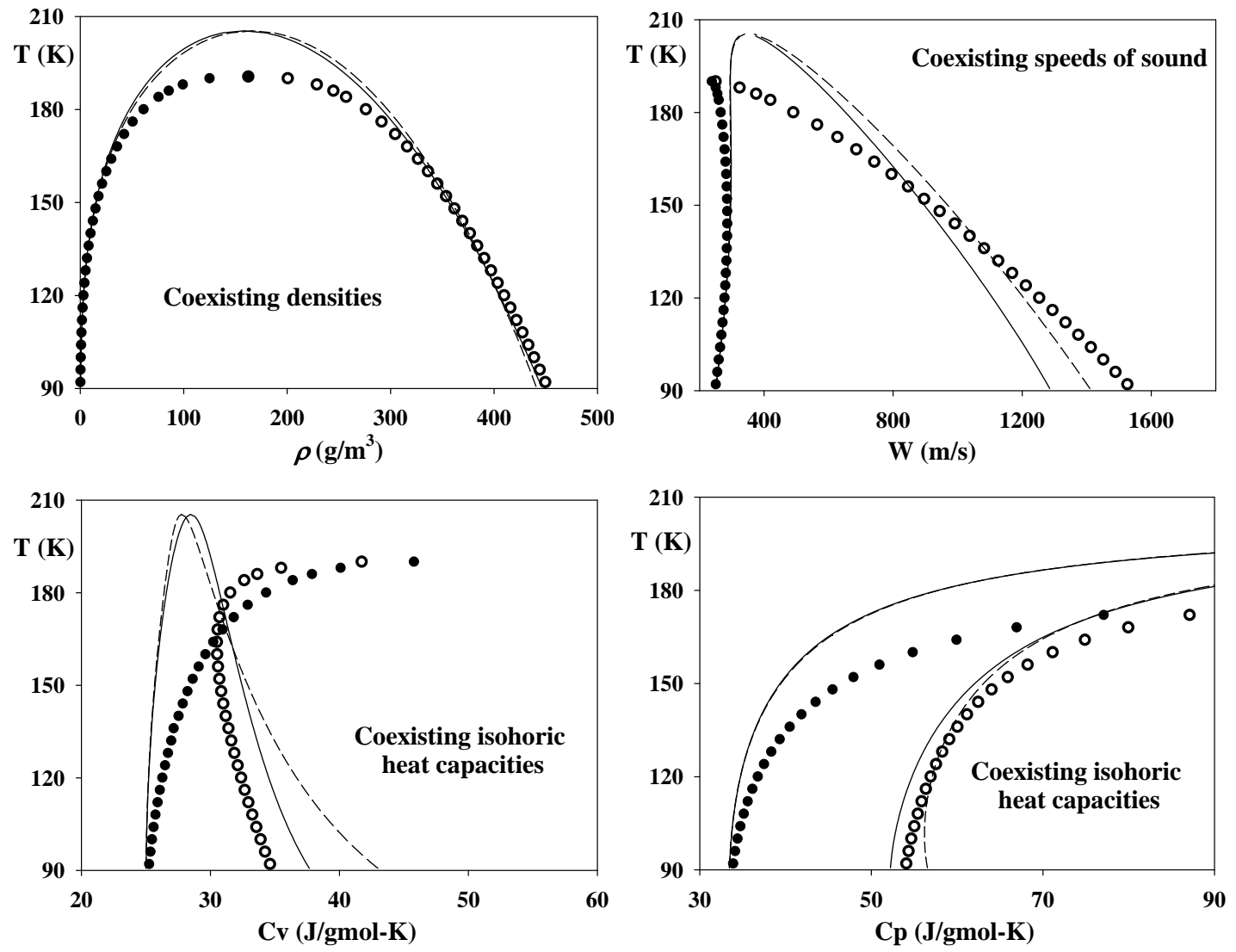

Fig. (3). Coexisting densities, speeds of sound, isochoric and isobaric heat capacities of methane. Experimental data [18]: 0 - liquid phase, - - vapor phase. Solid lines - SAFT-VR-Mie; dotted lines - SAFT of Chapman et al. 

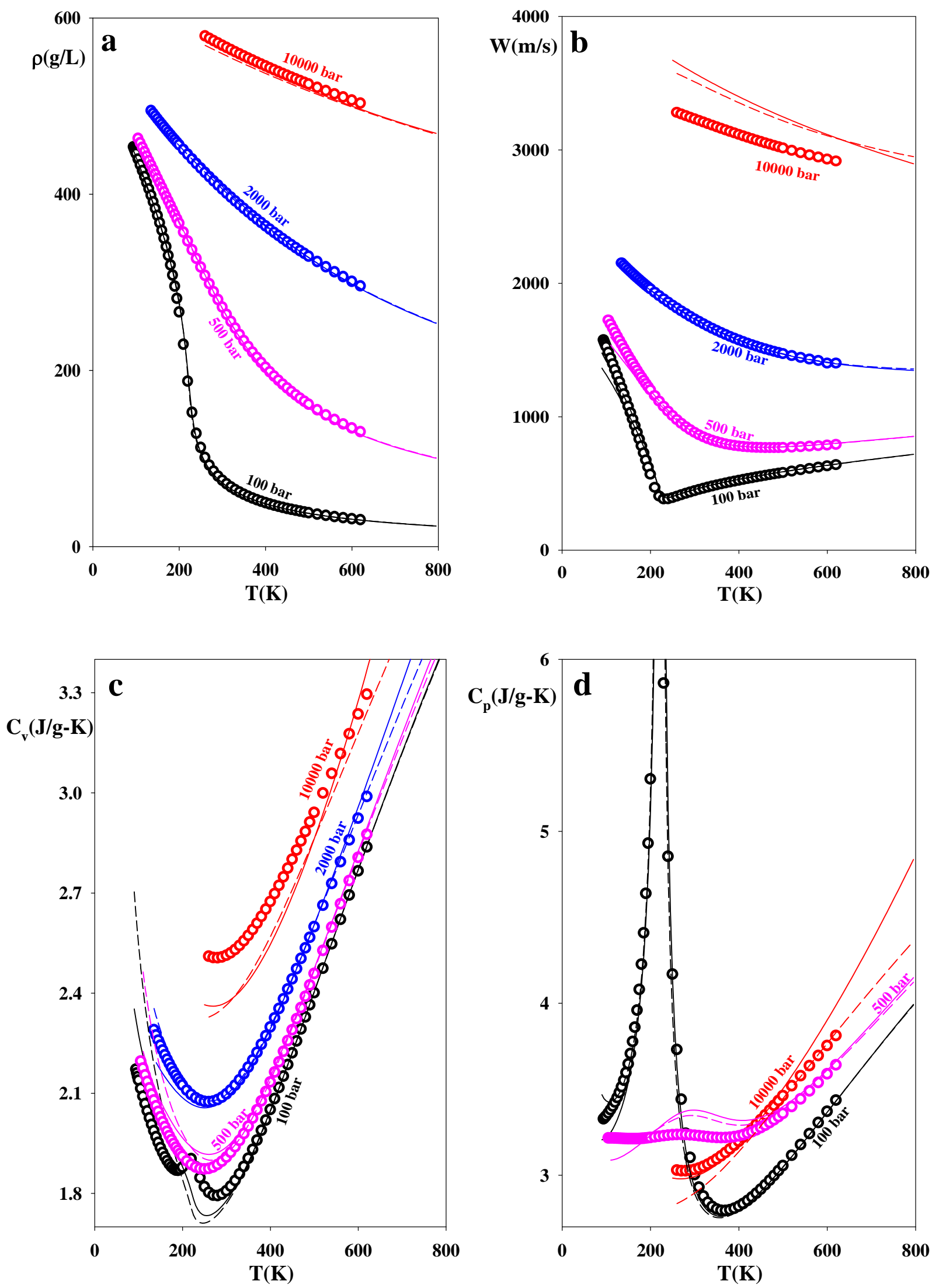

Fig. (4). High pressure densities, speeds of sound, isochoric and isobaric heat capacities of methane. Experimental data [18]: $\bigcirc-100$ bar, -500 bar, $\circ-2000$ bar, $\circ-10,000$ bar. Solid lines - SAFT-VR-Mie; dotted lines - SAFT of Chapman et al. 

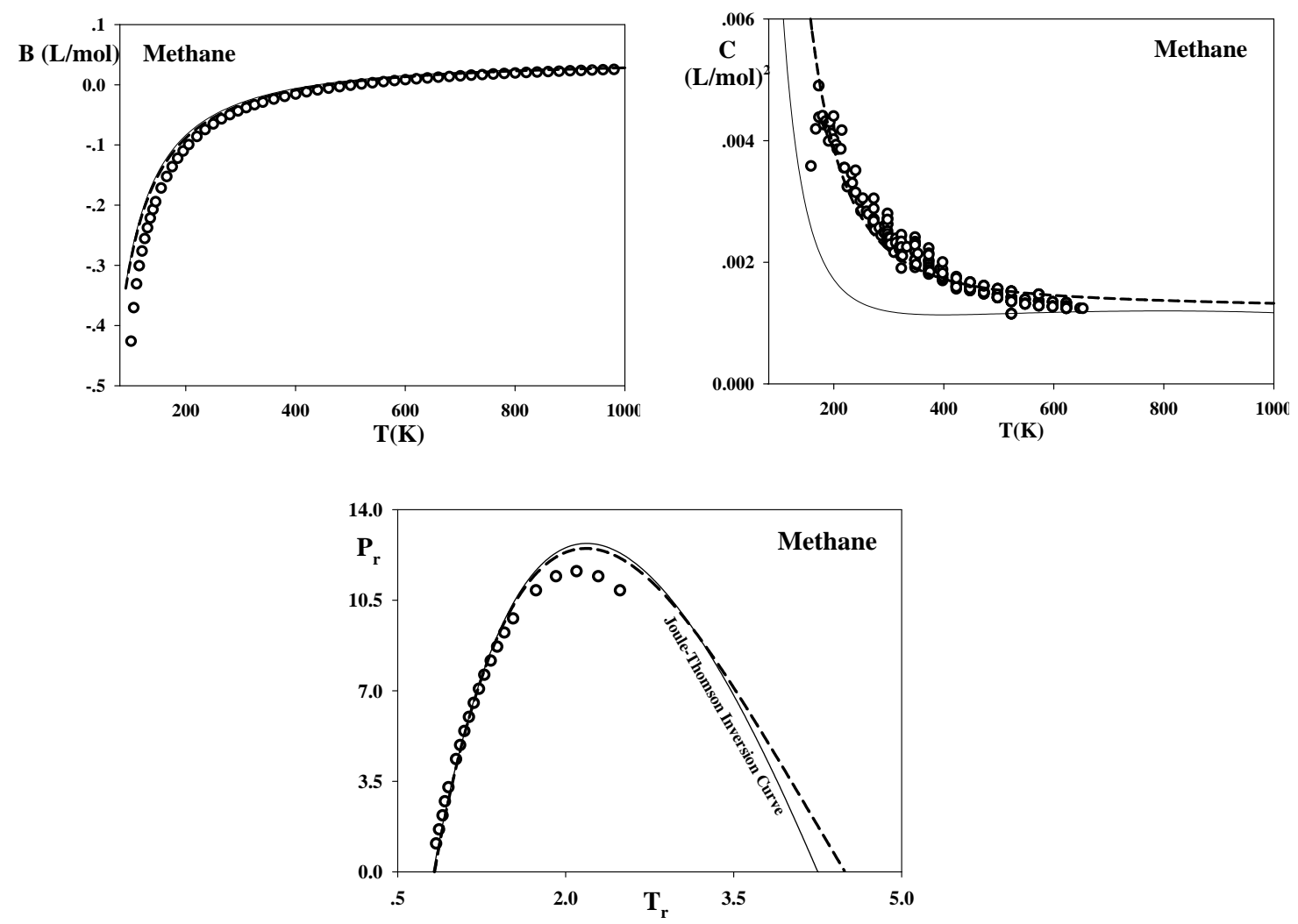

Fig. (5). Virial coefficients and Joule-Thomson inversion curve of methane. o - experimental data [17,19,20]. Solid lines - SAFT-VR-Mie; dotted lines - SAFT of Chapman et al.
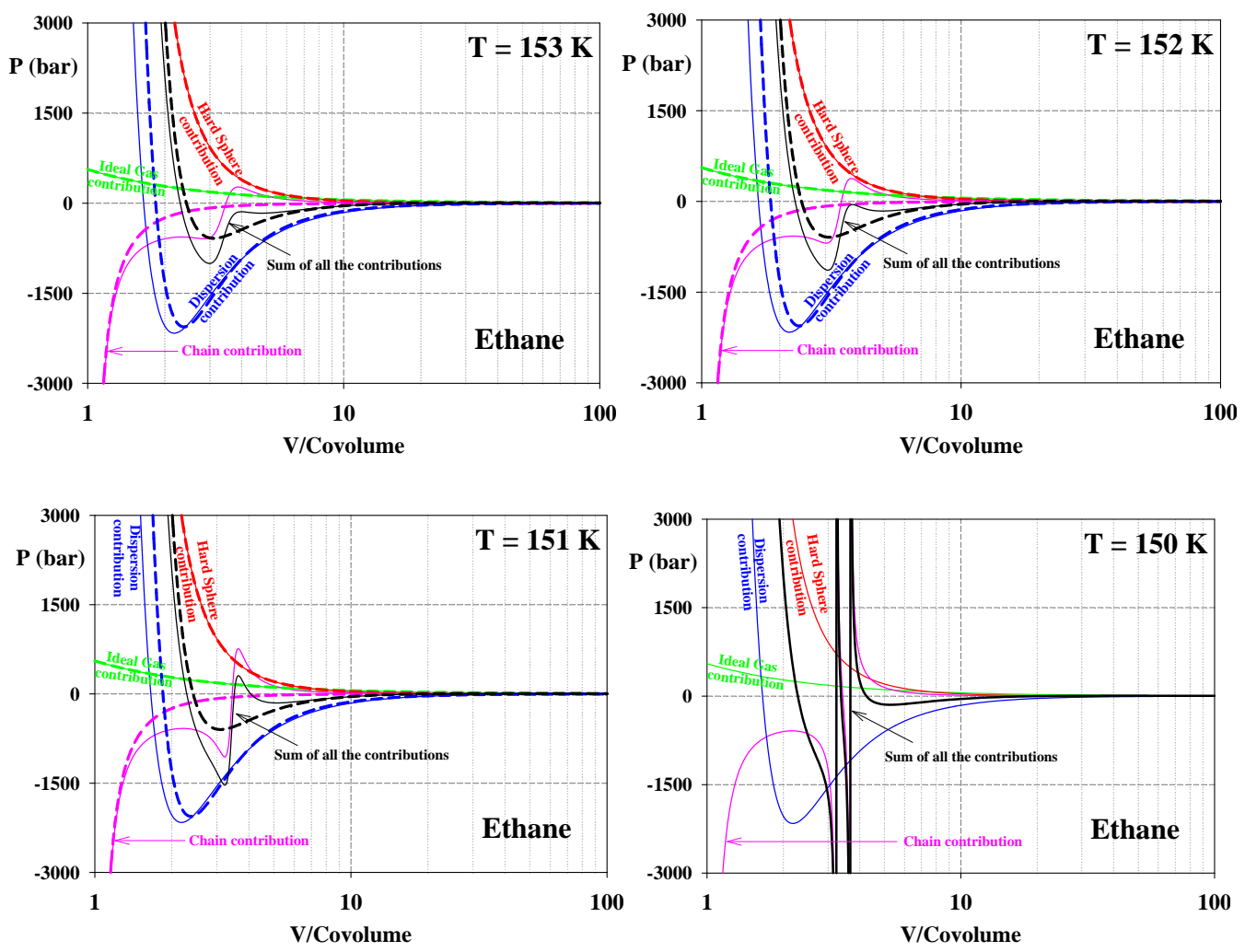

Fig. (6). Pressure contributions of different SAFT's parts for ethane at 150-153 K. Solid lines - SAFT-VR-Mie. Dotted lines - SAFT of Chapman's et al. 

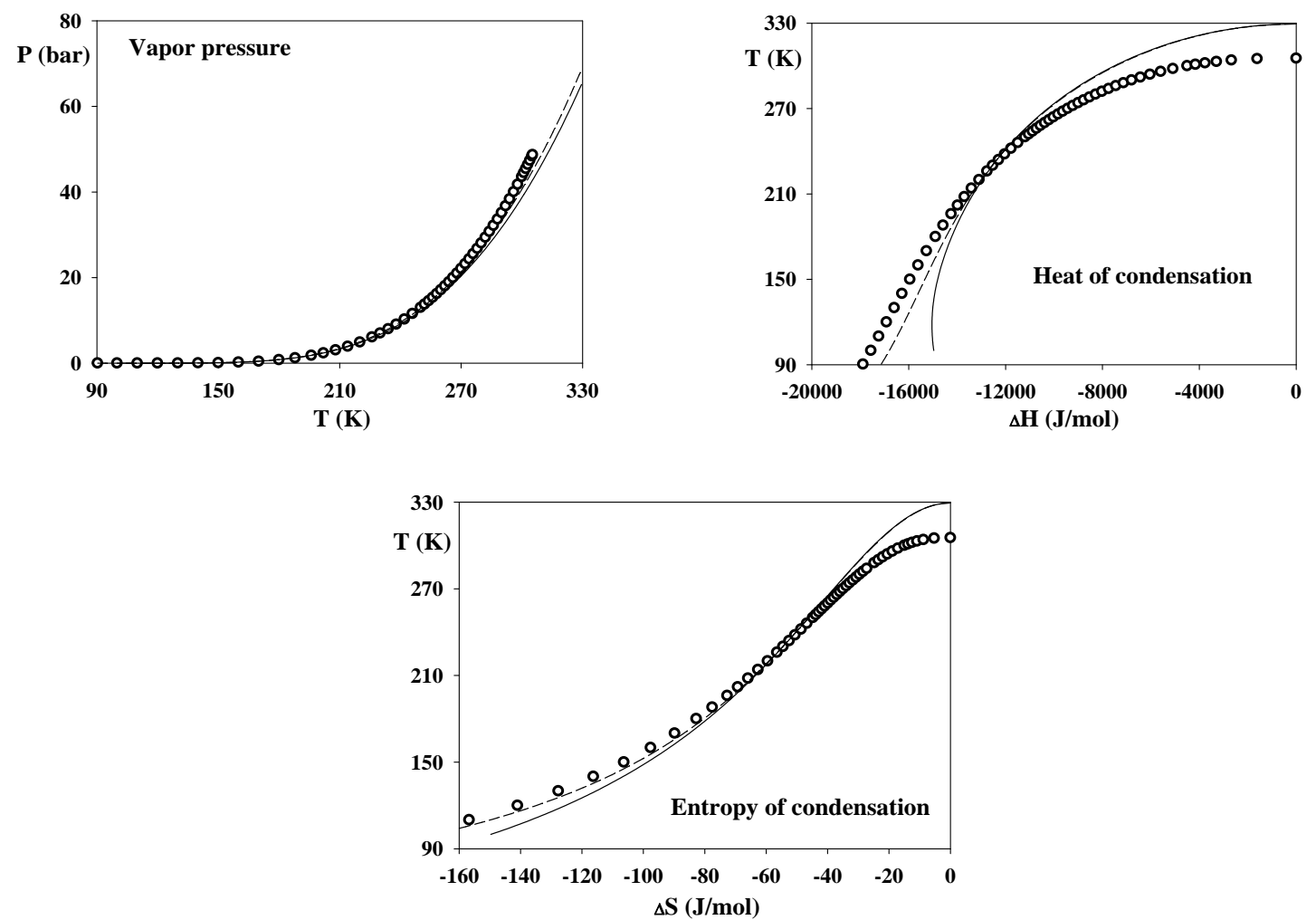

Fig. (7). Vapor pressure, enthalpy and entropy of condensation of ethane. o - experimental data [21]. Solid lines - SAFT-VR-Mie; dotted lines - SAFT of Chapman et al.
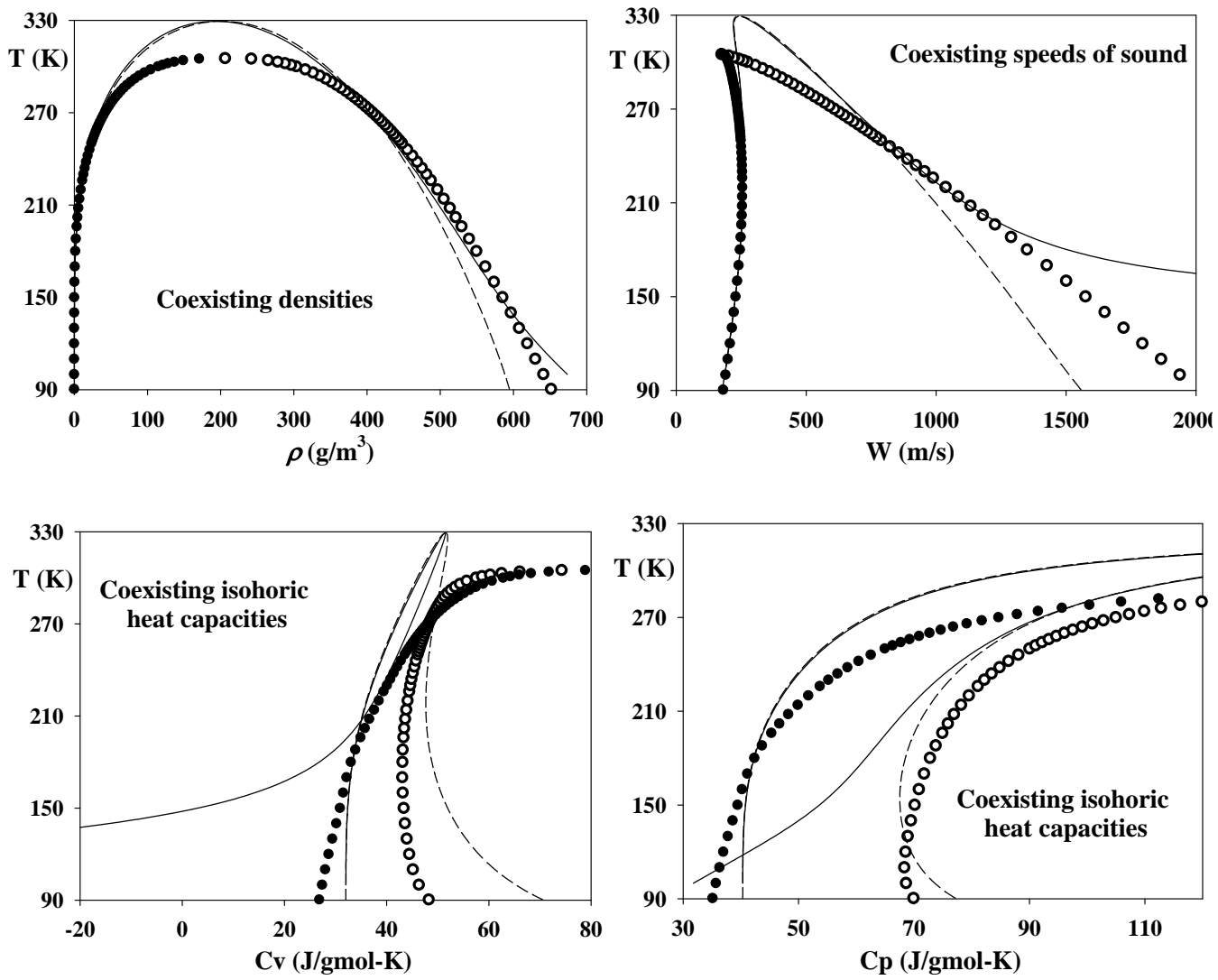

Fig. (8). Coexisting densities, speeds of sound, isochoric and isobaric heat capacities of ethane. Experimental data [21]: $0-$ liquid phase, - - vapor phase. Solid lines - SAFT-VR-Mie; dotted lines - SAFT of Chapman et al. 
more the HS contributions of both models are nearly identical and the SAFT-VR-Mie's dispersion contribution is a bit smaller. However the chain term yet yields some unexpected results. The figure demonstrates development of the previously unnoticed numerical pitfall behaviour. In particular, it might be seen that the SAFT-VR-Mie's chain term at certain temperatures might generate an additional fictitious covolume and the artificial phase instability. As a result, the model might predict the negative values of the isochoric heat capacities and the unrealistic results for sound velocities (see Figs. 8, 9). At the same time, outside the numerical failure region, the SAFT-VR-Mie's chain term contributes to a better estimation of data. In particular, Figs. (6-10) demonstrate its over-all advantage over the SAFT of Chapman et al. at the temperatures above $\sim 200 \mathrm{~K}$.
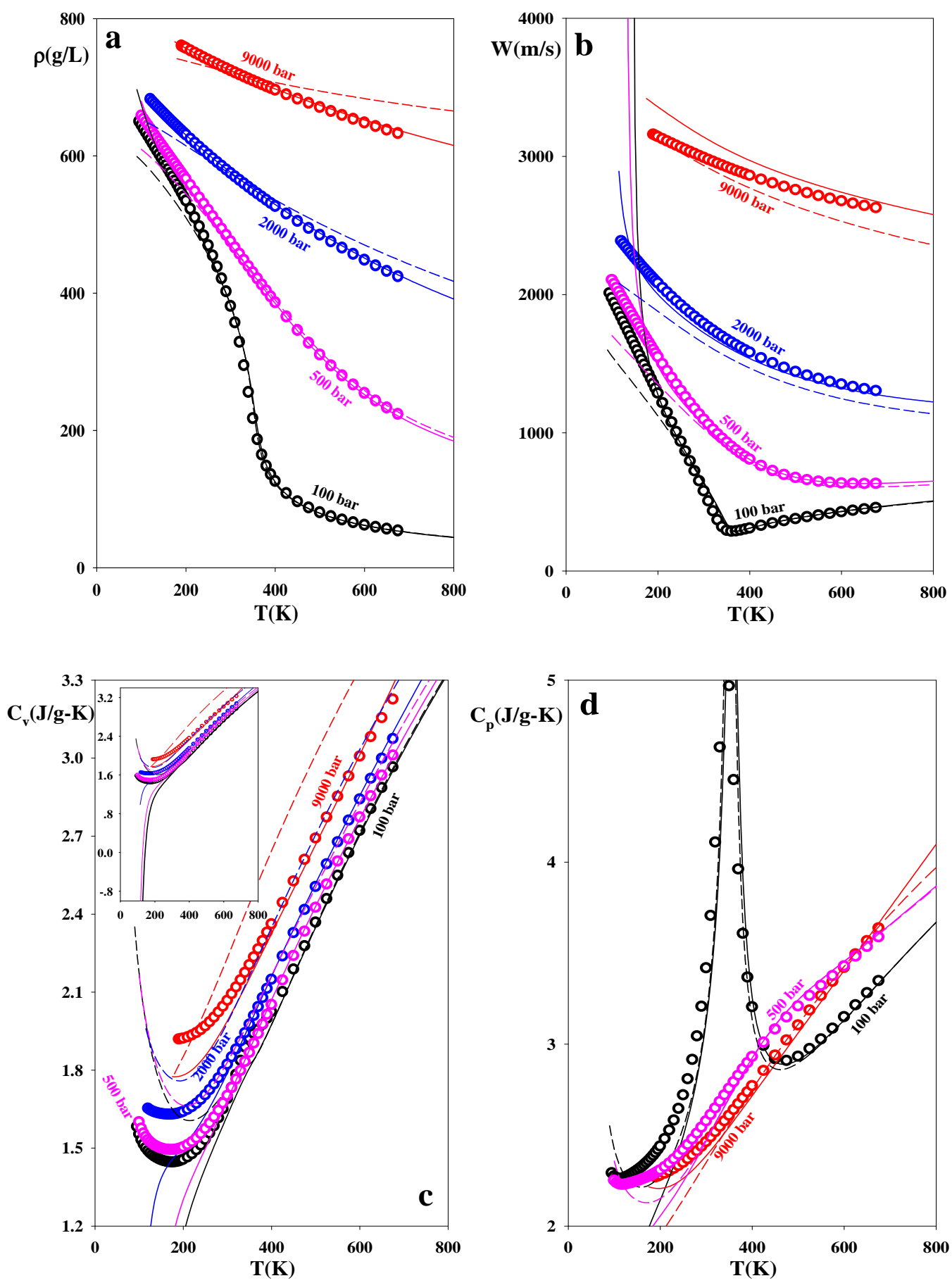

Fig. (9). High pressure densities, speeds of sound, isochoric and isobaric heat capacities of ethane. Experimental data [21]: $\bigcirc-100$ bar, $\circ-500$ bar, ○-2000 bar, ○- 9000 bar. Solid lines - SAFT-VR-Mie; dotted lines - SAFT of Chapman et al. 

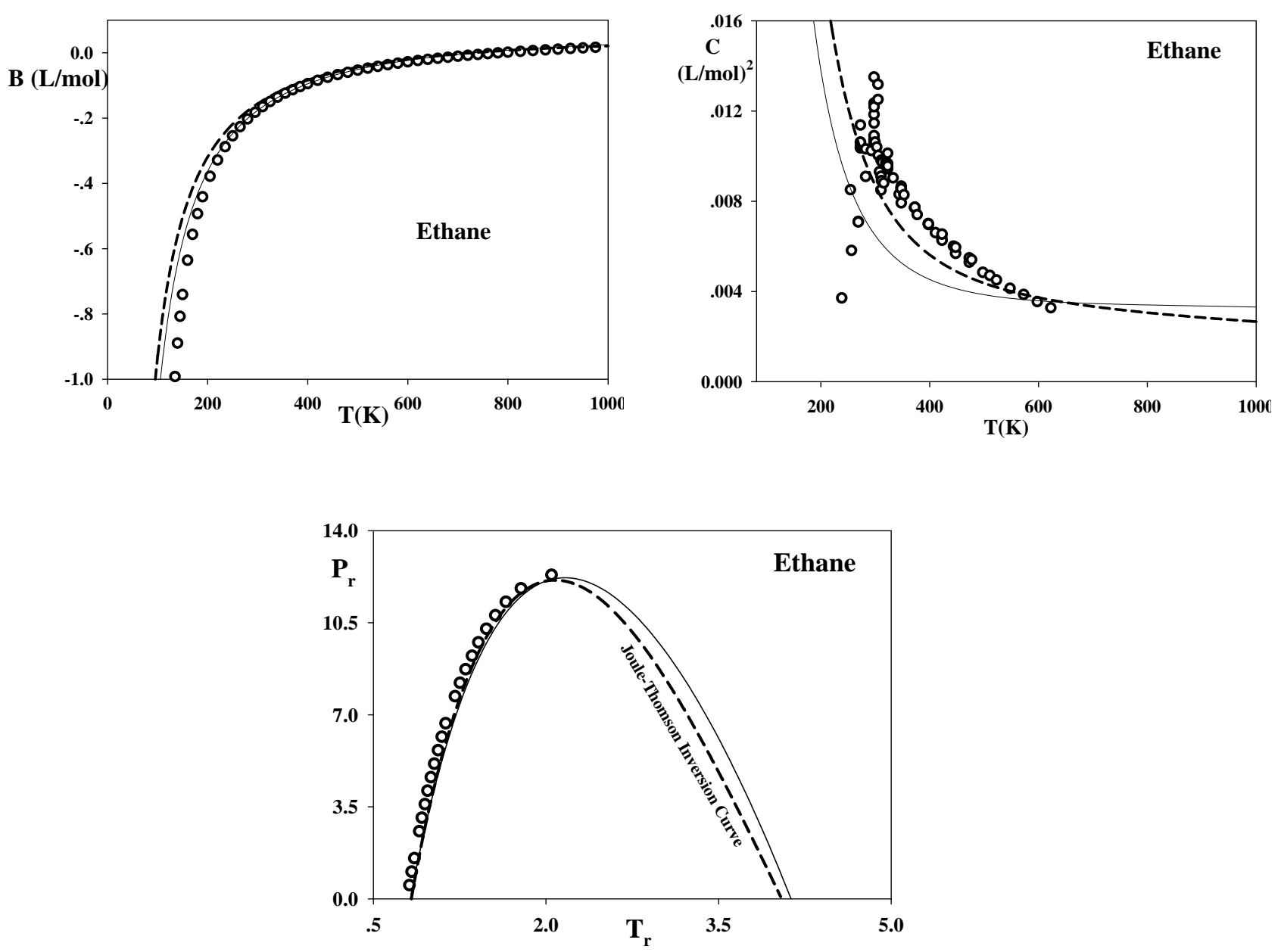

Fig. (10). Virial coefficients and Joule-Thomson inversion curve of ethane. $\odot$ - experimental data [17,19,20]. Solid lines - SAFT-VR-Mie; dotted lines - SAFT of Chapman et al.

Numerical analysis of Equation (13c) indicates that the numerical pitfall described above is caused by an artificial covolume generated by its last term and it does not seem to be related to the theoretical background of the model [22]. This numerical pitfall might easily be removed by reducing the numerical value of the last term of Equation (13c), for example as follows:

$A^{\text {chain }}=R T(1-m) \ln$

$\left[\frac{1-\eta / 2}{(1-\eta)^{3}}+\frac{\beta}{4}\left\{\frac{\partial a_{1}^{M}}{\partial \eta}-\frac{C \lambda_{1}}{3 \eta} a_{1}^{S}\left(\lambda_{1}\right)+\frac{3 C \lambda_{2}}{10 \eta} a_{1}^{S}\left(\lambda_{2}\right)\right\}\right]$

Implementation of Equation (27) instead of Equation (13c) makes the results of SAFT-VR-Mie remarkable similar to the SAFT of Chapman et al.

\section{CONCLUSIONS}

This study demonstrates that the advanced theoretical basis and the consequential numerical complexity do not always guarantee the success of EOS models in predicting the experimental thermodynamic property data. In particular, it is demonstrated that the predictive capabilities of the advanced version of SAFT, namely SAFT-VR-Mie in the case of the spherical non-polar molecules such as methane might in fact be similar to the modeling capacity of the much simpler version of SAFT, namely the SAFT of Chapman et al. Although SAFT-VR-Mie might have a doubtless advantage in predicting the data of non-spherical molecules, once again there is a price to pay for the excessive model's complexity. In particular, such complexity might result in appearance of undesired numerical pitfalls. Thus the present study reveals a previously unnoticed kind of numerical pitfalls, yet generated by the chain term of the SAFT-VR-Mie EOS. A possible way of avoiding the numerical pitfall under consideration is proposed.

\section{CONFLICT OF INTEREST}

None declared.

\section{ACKNOWLEDGMENTS}

Acknowledgment is made to the Donors of the American Chemical Society Petroleum Research Fund for support of this research, grant $\mathrm{N}^{\circ} \mathrm{PRF} \# 47338-\mathrm{B} 6$. 


\section{SYMBOLS}

\begin{tabular}{|c|c|c|}
\hline$A$ & $=$ & Helmholtz free energy \\
\hline$B$ & $=$ & Second virial coefficient \\
\hline$C$ & $=$ & Third virial coefficient \\
\hline$C_{V}$ & $=$ & Isochoric heat capacity \\
\hline$C_{P}$ & $=$ & Isobaric heat capacity \\
\hline$m$ & $=$ & Effective number of segments \\
\hline$H$ & $=$ & Enthalpy \\
\hline$M_{w}$ & $=$ & Molecular weight \\
\hline$N_{A v}$ & $=$ & Avogadro's number \\
\hline$P$ & $=$ & Pressure \\
\hline$R$ & $=$ & Universal gas constant \\
\hline$S$ & $=$ & Entropy \\
\hline$T$ & $=$ & Temperature \\
\hline$v$ & $=$ & Molar volume \\
\hline$W$ & $=$ & Speed of sound \\
\hline 7 & $=$ & Compressibility \\
\hline
\end{tabular}

\section{Greek letters}

$\lambda_{1}, \lambda_{2}=$ Coefficients of the Mie's potential

$\eta \quad=$ Reduced density

$\varepsilon k=$ Segment energy parameter divided by Boltzmann's constant

$\theta(\mathrm{T})=$ Temperature dependence of reduced density

$\rho=$ Density

$\sigma=$ Lennard-Jones temperature-independent segment diameter $(\AA)$

\section{Subscripts}

$\mathrm{c} \quad=$ Critical state

\section{Superscripts}

res $\quad=$ Residual property

\section{Abbreviations}

EOS $=$ Equation of state

$\mathrm{HS}=$ Hard sphere

SAFT $=$ Statistical association fluid theory

\section{REFERENCES}

[1] Y. S. Wei, R. J. Sadus, "Equations of state for the calculation of fluid-phase equilibria", AIChE J., vol. 46, pp. 169-196, January 2000.

[2] E. A. Müller, K. E. Gubbins, "Molecular-based equations of state for associating fluids: a review of SAFT and related approaches", Ind. Eng. Chem. Res., vol. 40, pp. 2193-2211, July 2001.
[3] I. G. Economou, "Statistical associating fluid theory: a successful model for the calculation of thermodynamic and phase equilibrium properties of complex fluid mixtures", Ind. Eng. Chem. Res., vol. 41, pp. 953-962, March 2002.

[4] S. P. Tan, H. Adidharma, M. Radosz, "Recent advances and applications of statistical associating fluid theory", Ind. Eng. Chem. Res., vol. 47, pp. 8063-8082, December 2008.

[5] G. M. Kontogeorgis, G. K. Folas, Thermodynamic Models for Industrial Applications. From Classical and Advanced Mixing Rules to Association Theories. New York: John Wiley \& Sons, 2010.

[6] A. Gil-Villegas, A. Galindo, P. J. Whitehead, S. J. Mills, G. Jackson, A. N. Burgess, "Statistical associating fluid theory for chain molecules with attractive potentials of variable range", $J$. Chem. Phys., vol. 106, pp. 4168-4186, March 1997.

[7] T. Lafitte, D. Bessières, M. M. Piñeiro, J.-L. Daridon, "Simultaneous estimation of phase behavior and second-derivative properties using the statistical associating fluid theory with variable range approach", J. Chem. Phys., vol. 124, pp. 024509/116, January 2006.

[8] T. Lafitte, M. M. Piñeiro, J.-L. Daridon, D. Bessières, "A comprehensive description of chemical association effects on second derivative properties of alcohols through a SAFT-VR approach", J. Phys. Chem. B, vol. 111, pp. 3447-3461, March 2007.

[9] T. Lafitte, F. Plantier, M. M. Piñeiro, J.-L. Daridon, D. Bessières, "Accurate global thermophysical characterization of hydrofluoroethers through a statistical associating fluid theory variable range approach, based on new experimental high-pressure volumetric and acoustic data", Ind. Eng. Chem. Res., vol. 46, pp. 6998-7007, June 2007.

[10] M. Khammar, J. Shaw, "Speed of sound prediction in 1-n-alcohol+ n-alkane mixtures using a translated SAFT-VR-Mie equation of state", Fluid Phase Equilib., vol. 288, pp. 145-154, January 2010.

[11] I. Polishuk, "Addressing the issue of numerical pitfalls characteristic for SAFT EOS models", Fluid Phase Equilib., vol. 301, pp. 123-129, January 2011.

[12] N. Koak, Th. W. de Loos, R. A. Heidemann, "Effect of the power series dispersion term on the pressure-volume behavior of statistical associating fluid theory", Ind. Eng. Chem. Res., vol. 38, pp. 1718-1722, February 1999.

[13] L.Yelash, M. Müller, W. Paul, K. Binder, "A global investigation of phase equilibria using the perturbed-chain statisticalassociating-fluid-theory approach", J. Chem. Phys., vol. 123, pp. 14908/1-15, July 2005.

[14] R. Privat, R. Gani, J.-N. Jaubert, "Are safe results obtained when the PC-SAFT equation of state is applied to ordinary pure chemicals?" Fluid Phase Equilib., vol. 295, pp. 76-92, August 2010.

[15] I. Polishuk, "About the numerical pitfalls characteristic for SAFT EOS models", Fluid Phase Equilib. vol. 298, pp. 67-74, November 2010.

[16] W. G. Chapman, K. E. Gubbins, G. Jackson, M. Radosz, "New reference equation of state for associating liquids", Ind. Eng. Chem. Res., vol. 29, pp. 1709-1721, February 1990.

[17] R. L. Rowley, W. V. Wilding, T. E. Daubert, R. P. Danner, M.E. Adams, Physical and Thermodynamic Properties of Pure Chemicals: DIPPR: Data Compilation. New-York: Taylor \& Francis, 2010.

[18] U. Setzmann, W. Wagner, "A new equation of state and tables of thermodynamic properties for methane covering the range from 'the melting line to $625 \mathrm{~K}$ at pressures up to $1000 \mathrm{MPa}$, J. Phys. Chem. Ref. Data, vol. 20, pp. 1061-1155, June 1991.

[19] D. W. Green, R. H. Perry, Perry's Chemical Engineers' Handbook (8th Edition). New-York: McGraw-Hill, 2008.

[20] J. H. Dymond, K. N. Marsh, R. C. Wilhoit, K. C. Wong, Virial Coefficients of Pure Gases and Mixtures. Landolt-Börnstein 
Numerical Data and Functional Relationships in Science and Technology New Series Group IV: Physical Chemistry Vol. 21. Berlin: Springer-Verlag, 2002.

[21] D. Bücker, W. Wagner, "A reference equation of state for the thermodynamic properties of ethane for temperatures from the melting line to $675 \mathrm{~K}$ and pressures up to $900 \mathrm{MPa}$ ", J. Phys. Chem. Ref. Data, vol. 35, pp. 205-266, January 2006.
[22] G. Galliero, T. Lafitte, D. Bessieres, C. Boned, "Thermodynamic properties of the Mie $n-6$ fluid: A comparison between statistical associating fluid theory of variable range approach and molecular dynamics results", J. Chem. Phys., vol. 127, pp. 184506/1184506/12, May 2007.

(C) Kalikhman et al.; Licensee Bentham Open.

This is an open access article licensed under the terms of the Creative Commons Attribution Non-Commercial License

(http://creativecommons.org/licenses/by-nc/3.0/) which permits unrestricted, non-commercial use, distribution and reproduction in any medium, provided the work is properly cited. 